\title{
CXCR1 blockade selectively targets human breast cancer stem cells in vitro and in xenografts
}

\author{
Christophe Ginestier, ${ }^{1,2}$ Suling Liu,, ${ }^{1}$ Mark E. Diebel, ${ }^{1}$ Hasan Korkaya, ${ }^{1}$ Ming Luo, ${ }^{3}$ Marty Brown, ${ }^{1}$ \\ Julien Wicinski, ${ }^{2}$ Olivier Cabaud, ${ }^{2}$ Emmanuelle Charafe-Jauffret, ${ }^{2}$ Daniel Birnbaum, ${ }^{2}$ \\ Jun-Lin Guan, ${ }^{3}$ Gabriela Dontu, ${ }^{1}$ and Max S. Wicha ${ }^{1}$

\begin{abstract}
${ }^{1}$ University of Michigan Comprehensive Cancer Center, Department of Internal Medicine/Oncology, Ann Arbor, Michigan, USA. Université de la Méditerranée, Marseille, France. ²Division of Molecular Medicine and Genetics and Cell and Developmental Biology, Department of Internal Medicine, University of Michigan Medical School, Ann Arbor, Michigan, USA.
\end{abstract} \\ ${ }^{2}$ Centre de Recherche en Cancérologie de Marseille, Laboratoire d’Oncologie Moléculaire, UMR891 INSERM/Institut Paoli-Calmettes,
}

\begin{abstract}
Recent evidence suggests that breast cancer and other solid tumors possess a rare population of cells capable of extensive self-renewal that contribute to metastasis and treatment resistance. We report here the development of a strategy to target these breast cancer stem cells (CSCs) through blockade of the IL-8 receptor CXCR1. CXCR1 blockade using either a CXCR1-specific blocking antibody or repertaxin, a small-molecule CXCR1 inhibitor, selectively depleted the CSC population in 2 human breast cancer cell lines in vitro. Furthermore, this was followed by the induction of massive apoptosis in the bulk tumor population via FASL/FAS signaling. The effects of CXCR1 blockade on CSC viability and on FASL production were mediated by the FAK/AKT/ FOXO3A pathway. In addition, repertaxin was able to specifically target the CSC population in human breast cancer xenografts, retarding tumor growth and reducing metastasis. Our data therefore suggest that CXCR1 blockade may provide a novel means of targeting and eliminating breast CSCs.
\end{abstract}

\section{Introduction}

The cancer stem cell (CSC) concept has important implications for understanding carcinogenesis as well as for the development of cancer therapeutics. According to this concept, tumors are initiated and maintained by a cellular subcomponent that displays stem cell properties. These properties include self-renewal, which drives tumorigenesis, and differentiation (albeit aberrant), which contributes to tumor cellular heterogeneity. The existence of CSCs has been described in a variety of hematologic and solid tumors including those of the breast, brain, colon, pancreas, lung, liver, and head and neck (1). In addition to driving tumorigenesis, CSCs may contribute to tumor metastasis as well as to tumor recurrence after treatment (2). Several recent studies have questioned the rarity of tumor cells with stem cell properties and tumor-initiating capacity as well as assays used to access these cell populations $(3,4)$. Nevertheless, in vitro and animal models have demonstrated that breast CSCs are relatively resistant to both radiation and chemotherapy $(5,6)$. This preclinical evidence has been supported by clinical studies demonstrating that the percentage of breast CSCs increased after neoadjuvant chemotherapy (7-9). Furthermore, the resistance of chronic myelogenous leukemia stem cells to imatinib (Gleevec), a BCR-ABL inhibitor, indicates that CSCs may also be resistant to some molecularly targeted agents. These studies suggest that the development of more effective cancer therapies may require effective targeting of the CSC population.

Authorship note: Suling Liu and Mark E. Diebel contributed equally to this work.

Conflict of interest: Max S. Wicha has financial holdings and is a scientific advisor for OncoMed Pharmaceuticals. The remaining authors have declared that no conflict of interest exists.

Citation for this article: J Clin Invest. 2010;120(2):485-497. doi:10.1172/JCI39397.
One of the therapeutic strategies being pursued to target CSCs involves inhibition of self-renewal or survival pathways in these cells. These pathways include NOTCH, Hedgehog, and WNT (10). Such strategies may be limited by the role of these pathways in normal stem cell function, which could result in systemic toxicities from pathway inhibition.

In addition to intrinsic pathways regulating stem cell functions, normal and malignant stem cells are regulated by extrinsic signals generated in the microenvironment or CSC niche. In the breast, this niche is composed of immune cells, mesenchymal elements that include fibroblasts, endothelial cells, adipocytes, and extracellular matrix components (11). These components play an important role in normal breast development and carcinogenesis. If the cellular microenvironment plays an important role in the regulation of CSC growth and survival, then strategies aimed at interfering with these interactions represent a rational approach to target breast CSCs.

We have previously reported that cells with stem cell characteristics can be isolated from normal human mammary glands as well as from breast carcinomas by virtue of the cellular expression of aldehyde dehydrogenase (ALDH), as assessed by the ALDEFLUOR assay (12). In breast carcinomas, the ALDEFLUOR ${ }^{+}$phenotype shows partial overlap with the previously described CD $44^{+} \mathrm{CD} 24^{-}$ Lin $^{-}$CSC phenotype. We have used similar techniques to identify cellular hierarchies in a series of molecularly characterized breast cancer cell lines and demonstrated that these lines contained $\mathrm{ALDEFLUOR}^{+}$components that were both tumorigenic and metastatic in NOD/SCID mice (13). Gene expression profiling of the $\mathrm{ALDEFLUOR}^{+}$populations revealed overexpression of CXCR1, a receptor for the cytokine IL-8. CXCR1 expression was limited to a subpopulation of ALDEFLUOR ${ }^{+}$cells. Furthermore, addition of recombinant IL-8 increased the CSC population as well as increas- 
ing its propensity for invasion (13). IL-8 has previously been implicated in tumor metastasis in preclinical models of prostate cancers (14). Furthermore, tissue damage induced by chemotherapeutic agents may induce IL- 8 as part of the injury response. This suggests that strategies aimed at interfering with the IL-8/CXCR1 axis may be able to target CSCs, increasing the efficacy of current therapies.

In the present study, we used both in vitro assays and mouse models to examine the effects of CXCR1 blockade on the breast CSC population. Using CXCR1-blocking antibodies or repertaxin, a small-molecule CXCR1 inhibitor, we demonstrated that CXCR1 blockade selectively decreased the breast CSC population in vitro and in NOD/SCID xenograft models. We showed that CXCR1 blockade induced massive apoptosis in bulk tumor cells via a bystander effect mediated by FASL/FAS signaling. CXCR1 effects on CSC viability as well as FASL production were mediated by the focal adhesion kinase/AKT/forkhead transcription factor FKHRL1 (FAK/AKT/FOXO3A) pathway. Furthermore, administration of repertaxin retarded tumor growth and reduced the development of systemic breast cancer metastasis in NOD/SCID mice.

\section{Results}

CXCR1 expression subdivides CSC populations. The elucidation of cell signaling pathways that regulate CSCs may lead to the identification of novel therapeutic targets in this cell population. We recently identified a breast CSC signature based on gene expression profiling that contained several genes potentially involved in breast CSC regulatory pathways (13). Among the genes overexpressed in the breast CSC population, CXCR1, a receptor that binds the proinflammatory chemokine IL-8/CXCL8, appeared to be a promising candidate because recombinant IL-8 stimulated the self-renewal of breast CSCs (13). Using flow cytometry, we assessed CXCR1 protein expression in the breast CSC population using the ALDEFLUOR assay in the human breast cancer cell lines HCC1954, MDA-MB-453, and SUM159. We previously demonstrated that in these cell lines, cells with functional stem cell properties in NOD/SCID mouse xenografts were contained within the ALDEFLUOR ${ }^{+}$cell population (13). We confirmed that the $\mathrm{CXCR} 1^{+}$population, which represented less than $2 \%$ of the total population, was almost exclusively contained within the $\mathrm{ALDEFLUOR}^{+}$population (Figure 1A and Supplemental Table 1; supplemental material available online with this article; doi:10.1172/ JDI39397DS1). We also assessed expression of CXCR2, a receptor that can also bind IL-8/CXL8, although with reduced affinity compared with CXCR1. In contrast to CXCR $1^{+}$cells, CXCR2 ${ }^{+}$cells were equally distributed between the ALDEFLUOR ${ }^{+}$and ALDEFLUORpopulations (Figure 1A). To determine the hierarchical organization of the CSC population according to CXCR1 expression, we injected sorted $\mathrm{ALDEFLUOR}{ }^{+} \mathrm{CXCR} 1^{+}$and $\mathrm{ALDEFLUOR}{ }^{+} \mathrm{CXCR} 1^{-}$cell populations and injected them into NOD/SCID mice (Supplemental Figure 1). Both cell populations generated tumors. Tumor growth kinetics correlated with the latency and size of tumor formation and the number of cells injected. Interestingly, tumors generated by the $\mathrm{ALDEFLUOR}^{+} \mathrm{CXCR} 1^{+}$population reconstituted the phenotypic heterogeneity of the initial tumor upon serial passages, whereas the ALDEFLUOR ${ }^{+}$CXCR1- $1^{-}$population gave rise to tumors containing only ALDEFLUOR ${ }^{+} \mathrm{CXCR} 1^{-}$cells. These results suggest that the CSC cellular hierarchy is organized according to CXCR1 expression; however, both cell populations displayed similar tumorigenic capacity.

CXCR1 blockade decreases the breast CSC population in vitro. To evaluate the effect of CXCR1 blockade on the breast CSC population, we treated the 3 cell lines with $100 \mathrm{nM}$ repertaxin, a CXCR1/2 inhibi- tor (15). For SUM159 cells, after 3 days of treatment, we observed a 5 -fold reduction in the proportion of ALDEFLUOR ${ }^{+}$cells (Figure 1B). Interestingly, a similar effect was observed after treatment of SUM159 cells with an anti-CXCR1 blocking antibody. In contrast, no effect was observed after treatment with an anti-CXCR2 blocking antibody, which suggests that the effects of repertaxin on the $\mathrm{ALDEFLUOR}^{+}$population were mediated by CXCR1.

Prior data from breast tumors, as well as cell lines, demonstrated that cancer stem-like cells or cancer-initiating cells can also be isolated and propagated as tumorspheres in suspension culture (16). After 3 days of treatment with repertaxin or with the anti-CXCR1 blocking antibody, when cells were detached and cultured in suspension, we observed an 8-fold decrease in primary and secondary tumorsphere formation compared with controls. In contrast, antiCXCR2 blocking antibody had no effect on tumorsphere formation (Supplemental Figure 2).

Surprisingly, after 5 days of treatment with repertaxin, we observed a massive decrease in viability of the entire cell population as assessed by MTT assay, with only $3 \%$ of cells remaining viable (Figure 1C). Because similar results were observed with the anti-CXCR1 blocking antibody, but not the anti-CXCR2 blocking antibody, we conclude that this effect was dependent on CXCR1 blockade. This effect of repertaxin was delayed, with loss of cell viability beginning 3 days after treatment (Supplemental Figure 3). Repertaxin treatment induced a similar effect on the HCC1954 breast cancer cell line, whereas no effect was observed on MDA-MB-453 cells, which harbor a PTEN mutation (Supplemental Figures 2-4 and ref. 17).

Using a TUNEL assay, we stained SUM159 cells after 4 days of treatment with repertaxin and confirmed that the massive decrease in cell viability was caused by induction of apoptosis, with $36 \%$ apoptotic cells detected after repertaxin treatment (Figure 1D). These results suggest that CXCR1 blockade results in a decrease of the breast CSC population followed by induction of massive apoptosis in the remaining bulk tumor population.

CXCR1 blockade induces cell death in CXCR1- cells via a bystander effect. The observation that repertaxin or anti-CXCR1 blocking antibody induced massive cell death, despite the CXCR1 $1^{+}$population representing less than $2 \%$ of the total cell population, suggested that CXCR1 blockade in CXCR $1^{+}$cells induced CXCR1 ${ }^{-}$cell death via a bystander effect. To validate this hypothesis, we treated the sorted CXCR1 ${ }^{+}$and CXCR1- populations with repertaxin (Figure 1E). Repertaxin decreased cell viability in the CXCR1 ${ }^{+}$ population within 3 days, whereas no effect was observed in the CXCR1- population. We confirmed that repertaxin induced massive cell death in unseparated cells. Moreover, the effect of repertaxin on cell viability of the unseparated and CXCR $1^{+}$populations was dose dependent (Figure 1E). These results are consistent with repertaxin treatment targeting the $\mathrm{CXCR} 1^{+}$population, which in turn induces CXCR1- cell death via a bystander effect.

To determine whether this effect was mediated by a soluble factor induced by repertaxin, we collected conditioned medium from the $\mathrm{CXCR} 1^{+}$population after 3 days of repertaxin treatment and dialyzed this medium using a membrane with 3.5 $\mathrm{kDa}$ exclusion in order to remove repertaxin from the medium while retaining molecules larger than $3.5 \mathrm{kDa}$. The dialyzed conditioned medium induced a massive decrease in cell viability in both CXCR1- and unseparated populations, but not in the $\mathrm{CXCR}^{+}$population (Figure $1 \mathrm{~F}$ ). These results demonstrate that CXCR1 blockade in the $\mathrm{CXCR} 1^{+}$population induces cell death 
A
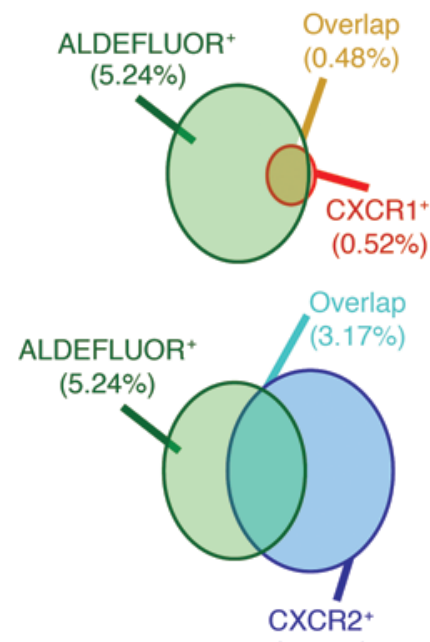

$(6.22 \%)$
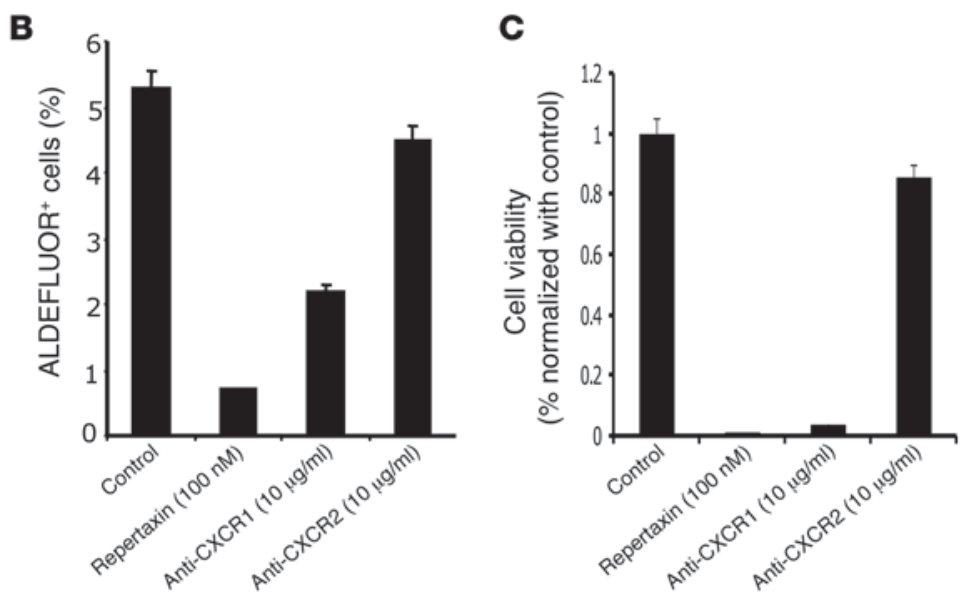
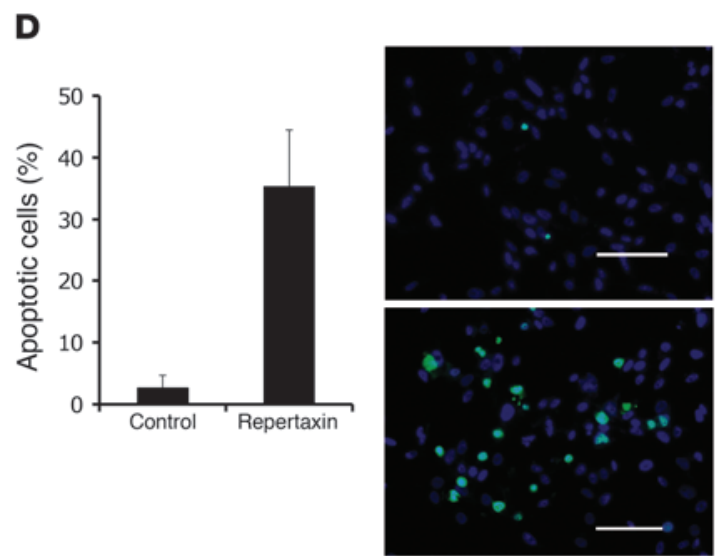

$\mathbf{E}$

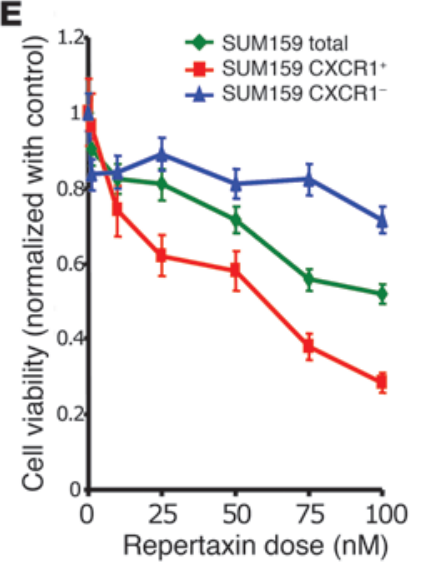

$\mathbf{F}$

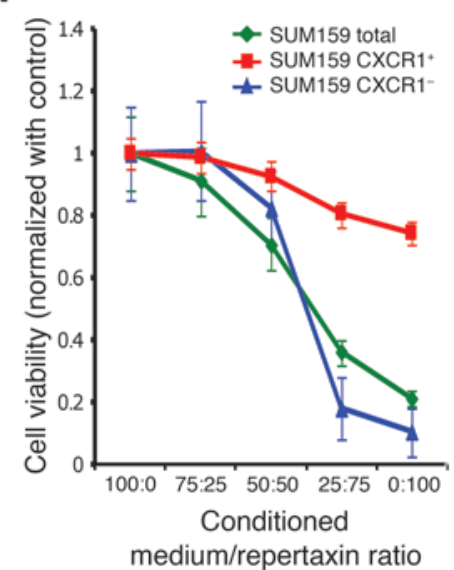

Figure 1

Effect of CXCR1 blockade on cell viability and on the ALDEFLUOR+ population in vitro. (A) Representation of the overlap between the ALDEFLUOR $^{+}$subpopulation and the CXCR1 $1^{+}$(top) or $\mathrm{CXCR}^{+}$(bottom) subpopulation of SUM159 cells. (B and C) SUM159 cells were cultured in adherent conditions and treated with repertaxin and 2 specific blocking antibodies for CXCR1 or CXCR2. After 3 days, the effect on cell viability and the CSC population was analyzed. A significant reduction of the ALDEFLUOR ${ }^{+}$population and cell viability was observed after treatment with repertaxin or anti-CXCR1 antibody, but not with anti-CXCR2 antibody. (D) After 4 days of treatment, the number of apoptotic cells was evaluated, and $36 \%$ of apoptotic cells (green) were detected in repertaxin-treated cells compared with controls, in which mostly viable cells (blue) were present. Scale bars: $100 \mu \mathrm{m}$. (E) To determine whether cell death was mediated via a bystander effect, CXCR1 ${ }^{+}$and CXCR1-populations were treated with various concentrations of repertaxin. A decrease in cell viability in CXCR $1^{+}$and unsorted populations were detected, whereas no effect was observed in the CXCR1- population. (F) Serial dilutions of dialyzed conditioned medium from CXCR1 ${ }^{+}$cells treated for 3 days with repertaxin was used to treat sorted $\mathrm{CXCR} 1^{+}, \mathrm{CXCR} 1^{-}$, or unsorted populations. After 2 days of treatment, a massive decrease in cell viability was observed in both CXCR1- and unseparated populations, whereas no effect was observed in the CXCR1+ population. Error bars represent mean \pm SD.

in the CXCR1- $1^{-}$population via a soluble nondialyzable factor. Although the CXCR1 $1^{+}$population is sensitive to repertaxin, it is resistant to the dialyzable death factor.

The bystander effect induced by CXCR1 blockade is mediated by FASL/ FAS signaling. FASL/FAS interaction is a well-known mediator of apoptosis associated with a bystander effect. It is activated in different physiologic states, such as mammary gland involution or conditions of tissue injury, including that induced by chemotherapy $(18,19)$. To evaluate the role of FASL/FAS interaction in mediating the apoptotic bystander effect induced by CXCR1 blockade, we measured the level of soluble FASL in the medium of repertaxin-treated SUM159 cells using ELISA. We observed a more than 5-fold increase of soluble FASL in the medium of cells treated for 4 days with repertaxin compared with untreated cells (Figure 2A). We confirmed the transcriptional regulation of FASL by repertaxin treatment through measuring FASL mRNA levels by RT-PCR (Figure 2B). We found a 4-fold increase in FASL mRNA in the repertaxin-treated cells compared with untreated cells. Similar results were observed after treatment with a FAS agonist that activates FAS signaling, which indicates that FASL is a target of FAS signaling that generates a positive feedback loop. Moreover, $100 \%$ of the SUM159 cells expressed FAS protein, as determined by flow cytometry (data not shown). Treatment of the SUM159 cells with the FAS agonist reproduced the killing effect observed with repertaxin treatment, with massive reduction in cell viability (Figure $2 \mathrm{C}$ ). Furthermore, the effect of repertaxin treatment on cell 


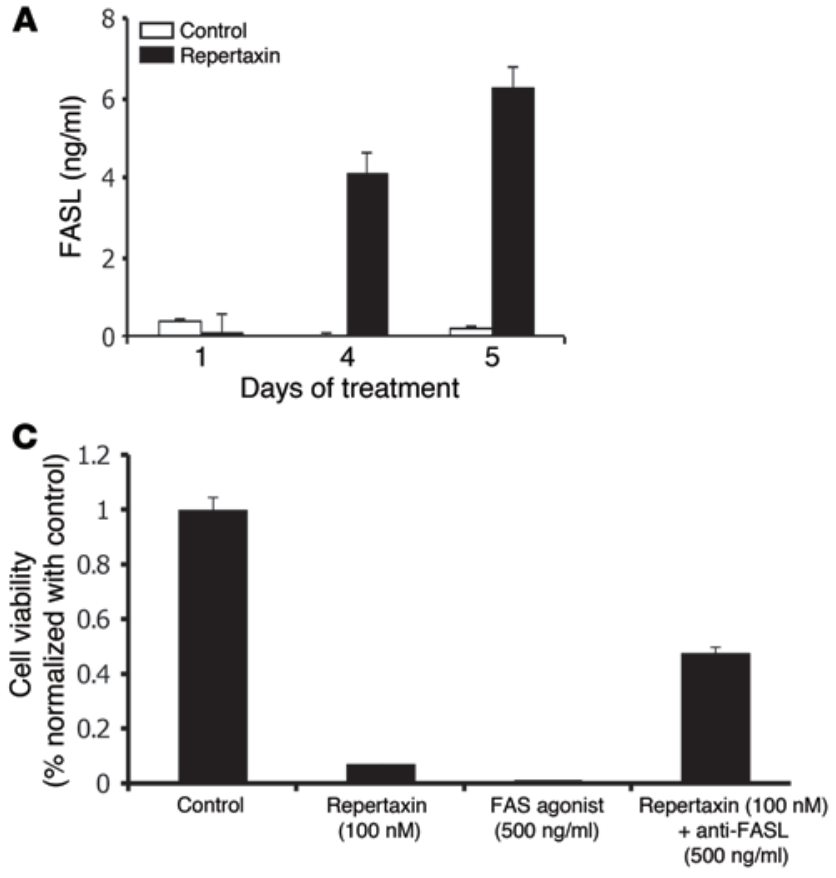

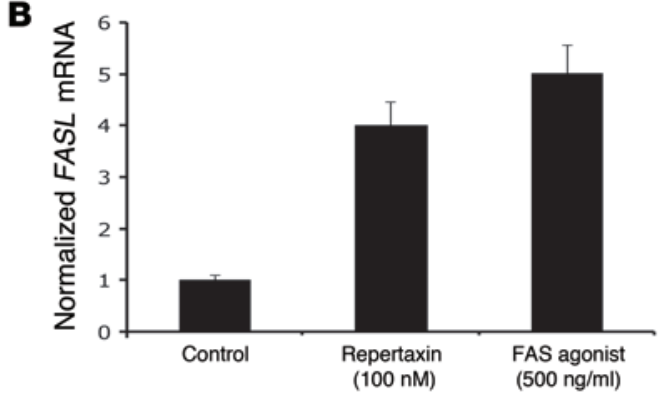

D

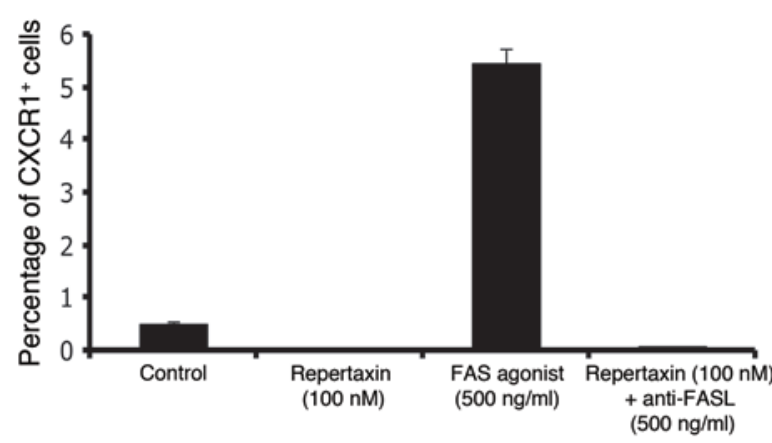

E

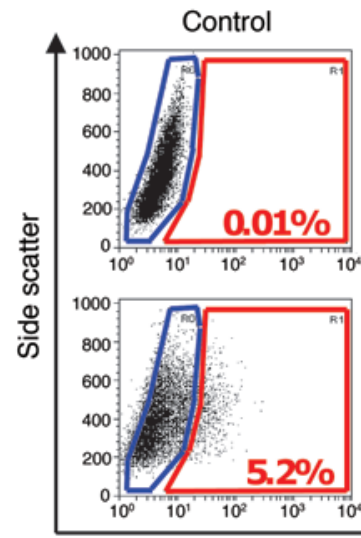

Repertaxin

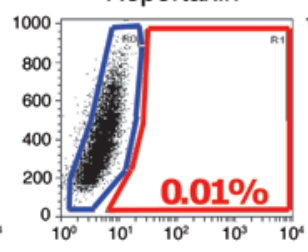

FAS agonist

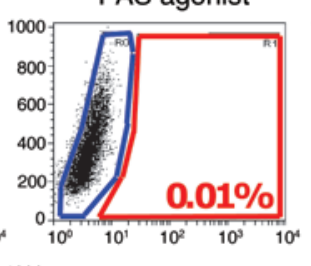

Repertaxin + anti-FASL
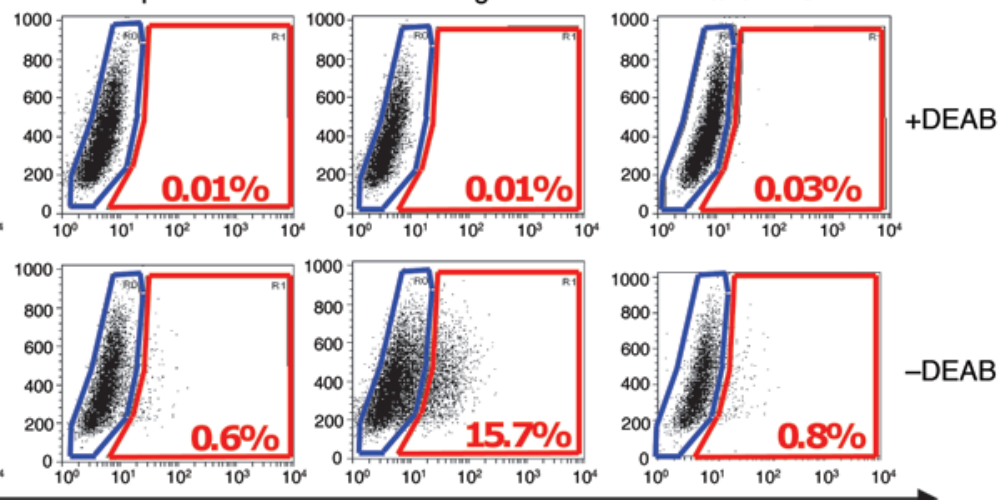
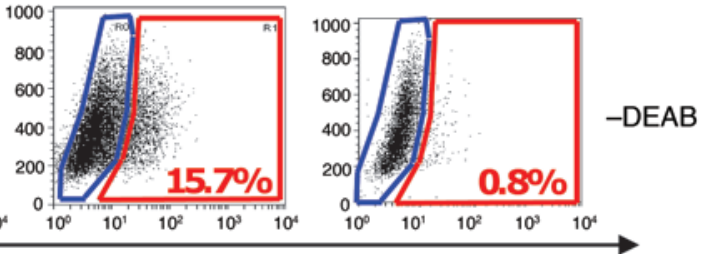

BAAA

\section{Figure 2}

Repertaxin treatment induces a bystander effect mediated by FASL/FAS signaling. (A) To determine whether the bystander killing effect induced by repertaxin treatment was mediated by FASL, we measured the level of soluble FASL in the medium using an ELISA assay. After 4 days of treatment, a greater than 4-fold increase of soluble FASL was detected in the medium of cells treated with repertaxin compared with untreated controls. (B) We measured the level of FASL mRNA by RT-PCR and confirmed the increase of FASL production after treatment with repertaxin. Similar results were observed after 4 days of treatment with a FAS agonist that activates FAS signaling, with a 5-fold increase of the FASL mRNA compared with control. (C) SUM159 cells were cultured in adherent conditions and treated with repertaxin alone or in combination with anti-FASL. Interestingly, cell growth inhibition induced by repertaxin treatment was partially rescued by addition of anti-FASL. Moreover, cells treated with a FAS agonist displayed cell growth inhibition similar to that of cells treated with repertaxin alone. (D and $\mathbf{E})$ The effect of repertaxin treatment, alone or in combination with anti-FASL, and FAS agonist treatment on the CXCR1 ${ }^{+}$and ALDEFLUOR ${ }^{+}$population was analyzed. The massive decrease in the $\mathrm{CXCR} 1^{+}$and $\mathrm{ALDEFLUOR}{ }^{+}$population induced by repertaxin treatment was not rescued by the anti-FASL, and treatment with FAS agonist produced 10- and 3-fold increases in the percent of the CXCR1+ and ALDEFLUOR+ populations, respectively. BAAA, BODIPY aminoacetaldehyde; DEAB, diethylaminobenzaldehyde. Error bars represent mean \pm SD.

viability was partially reversed by an anti-FASL blocking antibody, with $44 \%$ of cells remaining viable after treatment with repertaxin and anti-FASL antibody compared with $3 \%$ of cells treated with repertaxin alone (Figure $2 \mathrm{C}$ ). These results suggest that the massive cell death induced by repertaxin is the result of a bystander effect mediated by the FASL/FAS pathway.
Remarkably, treatment of SUM159 cells with the FAS agonist resulted in a 10- and 3 -fold increase in the percentage of CXCR1 ${ }^{+}$ and ALDEFLUOR ${ }^{+}$cells, respectively (Figure 2, D and E, and Supplemental Figure 5). Furthermore, the effects of repertaxin on the both populations were not rescued by anti-FASL (Figure 2, D and E). This suggests that the ALDEFLUOR ${ }^{+}$population that contains the 
CXCR $1^{+}$population - while directly sensitive to CXCR1 blockade, which in turn induces FASL production by these cells - is resistant to FASL/FAS proapoptotic signaling. In contrast, the ALDEFLUOR- bulk cell population does not express CXCR1, but is sensitive to FASL-mediated cell death.

Because FASL/FAS signaling plays an important role during mammary gland involution (19), we analyzed the effect of CXCR1 blockade on normal human mammary epithelial cells obtained from reduction mammoplasties. As observed in breast cancer cell lines, $\mathrm{CXCR}^{+}$normal mammary cells were almost exclusively contained within the ALDEFLUOR ${ }^{+}$population (Supplemental Figure 6). To determine whether IL-8 signaling is important in normal breast stem/progenitor function, we treated normal mammary epithelial cells cultured in suspension with human recombinant IL-8 and determined its effect on the CSC population, as measured by the formation of mammospheres (20). As shown in Supplemental Figure 6, addition of IL-8 increased the formation of primary and secondary mammospheres in a dose-dependent manner. This suggests that the IL-8/CXCR1 axis may be involved in the regulation of normal mammary stem/progenitor cell proliferation or self-renewal. Interestingly, treatment with repertaxin or the FAS agonist had no effect on the viability of normal mammary epithelial cells cultured in adherent conditions, even when high concentrations of repertaxin were used (500 nM; Supplemental Figure 4). However, as observed for breast cancer cell lines, we detected an increase of soluble FASL in the medium of normal mammary epithelial cells treated with repertaxin (Supplemental Figure 7). This observation may be explained by the absence of FAS expression in the normal epithelial cells cultured under these conditions (Supplemental Figure 7). This observation is consistent with the prior demonstration that FAS expression in the mammary gland occurs only during the involution process after lactation (19). In contrast to its lack of effect on the bulk population of normal mammary epithelial cells, repertaxin significantly decreased mammosphere formation by these cells (Supplemental Figure 7). Together, these results suggest that the IL-8/CXCR1 axis plays an important role in the regulation and the survival of normal and malignant mammary epithelial stem/progenitor cell populations. The ability to affect bulk cell populations via a FASL-mediated bystander effect may relate to the level of FAS expression in these cells.

CXCR1 blockade effects on CSCs are mediated by the FAK/AKT/ FOXO3A pathway. CXCR1 has previously been shown to act through a signal transduction pathway involving phosphorylation of the FAK, resulting in activation of AKT (21). To evaluate the impact of CXCR1 blockade on FAK and AKT activation, we measured the levels of FAK- and AKT-phosphorylated proteins by Western blot for the 3 breast cancer cell lines. In SUM159 and HCC1954 cells, we detected a decrease in FAK Tyr ${ }^{397}$ and AKT Ser ${ }^{473}$ phosphorylation in cells treated with repertaxin compared with untreated cells, which suggests that the effects of repertaxin may be mediated by the FAK/AKT pathway (Figure 3A and Supplemental Figure 8). Interestingly, the observation that MDA-MB-453 cells were resistant to repertaxin treatment may be explained by the presence of a PTEN mutation (919G>A) that activates the PI3K/AKT pathway (17). We did not detect any modification in FAK Tyr ${ }^{397}$ and AKT $\mathrm{Ser}^{473}$ phosphorylation after repertaxin treatment in the MDAMB-453 cell line (Supplemental Figure 8). To confirm a functional role of the FAK/AKT pathway in mediating the effects of the CXCR1 blockade, we used 2 viral constructs, 1 knocking down PTEN expression via a PTEN shRNA and the other leading to FAK overexpression. PTEN, through its lipid phosphatase, antagonizes PI3K/AKT signaling (22). As expected, PTEN knockdown resulted in AKT activation, as demonstrated by an increase of AKT Ser ${ }^{473}$ phosphorylation (Figure 3A and Supplemental Figure 8). Interestingly, PTEN knockdown blocked the effect of repertaxin treatment on FAK and AKT activity. FAK overexpression also blocked the effects of repertaxin and induced activation of FAK and AKT, measured by increased expression of FAK Tyr ${ }^{397}$ and AKT Ser ${ }^{473}$ phosphorylation. These results suggest that CXCR1 blockade effects are mediated by FAK/AKT signaling.

Using immunofluorescence staining of CXCR $1^{+}$cells, we confirmed that repertaxin treatment caused a dramatic decrease of phospho-FAK and phospho-AKT expression compared with untreated cells (Figure $3 \mathrm{~B}$ ). AKT is able to regulate FOXO3A activity via a phosphorylation event resulting in cytoplasmic FOXO3A sequestration (23). In contrast, the nonphosphorylated form of FOXO3A transits to the nucleus, where it acts as a transcription factor that regulates the synthesis of FASL (24). Because repertaxin induced cell death via a FASL-mediated bystander effect, we examined the effects of repertaxin on this signal transduction pathway by immunofluorescence staining. As shown in Figure 3B, FOXO3A was present in a cytoplasmic localization in untreated cells, but shuttled to the nucleus upon repertaxin treatment. This suggests that CXCR1 blockade induces FOXO3A activity through inhibition of the FAK/AKT pathway. Cells with PTEN deletion or FAK overexpression displayed a high level of phospho-FAK and phospho-Akt expression, detected by immunofluorescence, in both repertaxin-treated and untreated cells. Moreover, repertaxin treatment did not induce FOXO3A activation in cells with PTEN deletion or FAK overexpression, as shown by the cytoplasmic location of FOXO3A (Figure 3B).

As a consequence of the constitutive activation of the FAK/ AKT pathway, cells with PTEN deletion or FAK overexpression displayed resistance to repertaxin treatment. As shown in Figure 3C and Supplemental Figure 9, cells with PTEN deletion or FAK overexpression did not display any decrease in cell viability with repertaxin treatment. It has been proposed that AKT signaling plays a critical role in the biology of CSCs (25-27). Activation of the FAK/AKT pathway blocked the effects of repertaxin on the CSC populations, as shown by the maintenance of the ALDE$\mathrm{FLUOR}^{+}$populations after treatment with the inhibitor (Figure 3D). All these results support a model in which CXCR1 blockade directly affects the FAK/AKT/FOXO3A pathway. Repertaxin treatment inhibits AKT signaling, which is crucial for CSC activity, and subsequently induces a bystander effect on the bulk tumor cells mediated by CSC-generated FASL.

Docetaxel and FASL induce IL8, increasing the CSC population. Recent evidence suggests that breast CSCs are relatively resistant to chemotherapy and radiation and may contribute to tumor regrowth after therapy (5-7). The CSC concept suggests that significant improvements in clinical outcome will require effective targeting of the CSC population (2). Several factors are synthesized and secreted during the apoptotic process when the bulk tumor cells are targeted by chemotherapy. Among these factors, FASL is known to amplify chemotherapy effects by mediating a bystander killing effect (18). Interestingly, chemotherapy may also induce IL-8 production in injured cells. We confirmed that the commonly used chemotherapeutic agent docetaxel induced both IL 8 and FASL mRNA in SUM159 cells (Supplemental Figure 10). We also detected a 4-fold increase of IL8 mRNA after FAS agonist treat- 
A

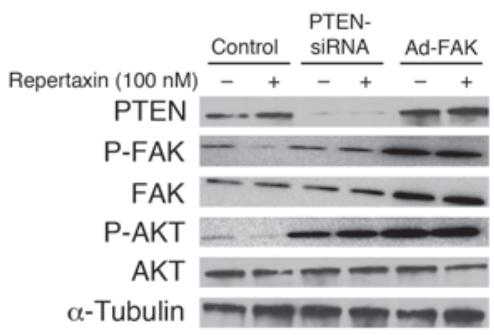

C

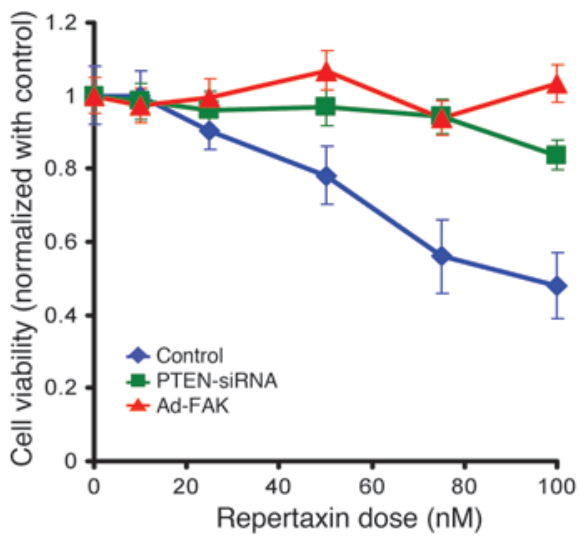

D

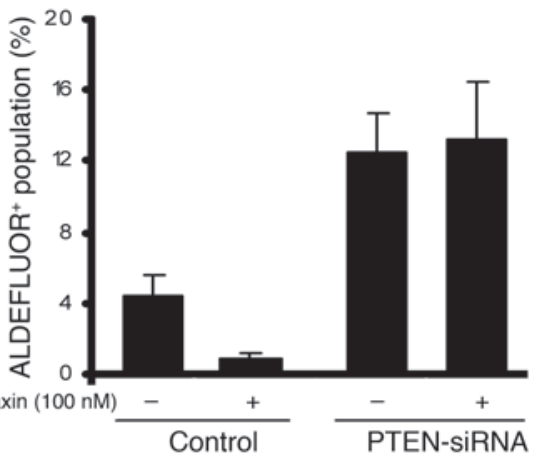

B

Repertaxin (100 nM)
Control

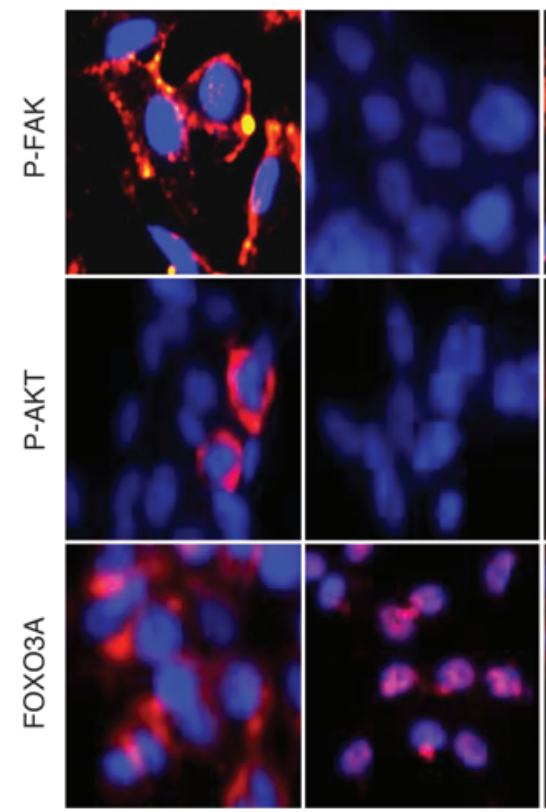

PTEN-siRNA

$\frac{\text { PTEN-SiRNA }}{-}$
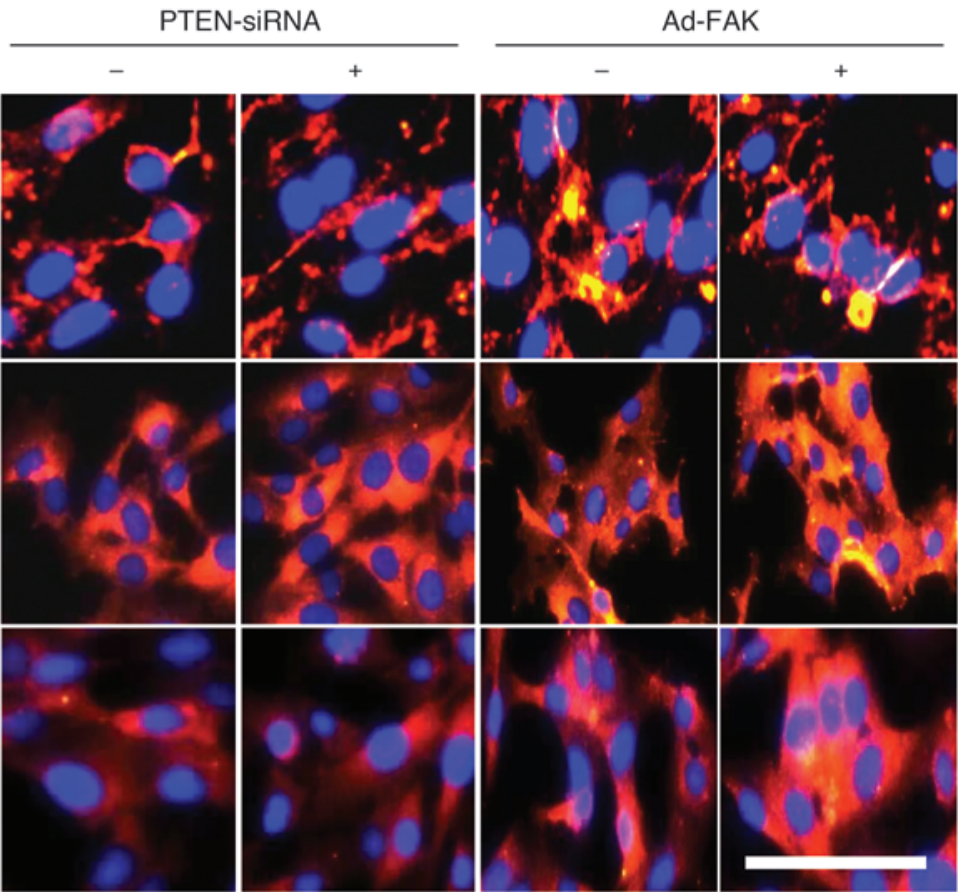

\section{Figure 3}

Effect of repertaxin treatment on FAK, AKT, and FOXO3A activation. To evaluate the effect of repertaxin treatment on CXCR1 downstream signaling, we used 2 different viral constructs, 1 knocking down PTEN expression via a PTEN-siRNA and the other leading to FAK overexpression (Ad-FAK). (A) Repertaxin treatment led to a decrease in FAK Tyr ${ }^{397}$ and AKT Ser ${ }^{473}$ phosphorylation, whereas PTEN deletion and FAK overexpression blocked the effect of repertaxin treatment on FAK and AKT activity. (B) Using immunofluorescence staining on CXCR1+ cells, we confirmed that repertaxin treatment caused a disappearance of phospho-FAK (membranous staining in red) and phospho-AKT expression (cytoplasmic staining in red). Immunofluorescence staining with anti-FOXO3A revealed a cytoplasmic location of FOXO3A (red) in the untreated cells, whereas repertaxin treatment induced a relocalization of FOXO3A to the nucleus. In contrast, cells with PTEN deletion or FAK overexpression displayed a high level of phospho-FAK, phospho-AKT, and cytoplasmic FOXO3A expression in both the repertaxin-treated and untreated cells. In all samples, nuclei were counterstained with DAPI (blue). Scale bar: $50 \mu \mathrm{m}$. (C and D) The effect of repertaxin on SUM159 PTEN-siRNA and SUM159 Ad-FAK cell viability and on the CSC population was assessed using MTT and ALDEFLUOR assays, respectively. After 3 days of treatment, cells with PTEN deletion or FAK overexpression developed resistance to repertaxin (C). Furthermore, repertaxin treatment did not alter the proportion of ALDEFLUOR+ SUM159 PTEN knockdown cells (D). Error bars represent mean \pm SD.

ment (Supplemental Figure 10), consistent with previous reports of IL-8 being a downstream target of FAS signaling (28). We have previously shown that IL-8 is able to regulate the CSC population (13). This may partially account for the increase in CSCs after chemotherapy (7). Furthermore, this suggests that the addition of repertaxin to cytotoxic chemotherapy may block this effect and target the CSC population.

Repertaxin treatment reduces the CSC population in tumor xenografts. To explore the effect of repertaxin treatment on tumor growth in vivo, we used SUM159 cells and 3 primary human breast cancer 

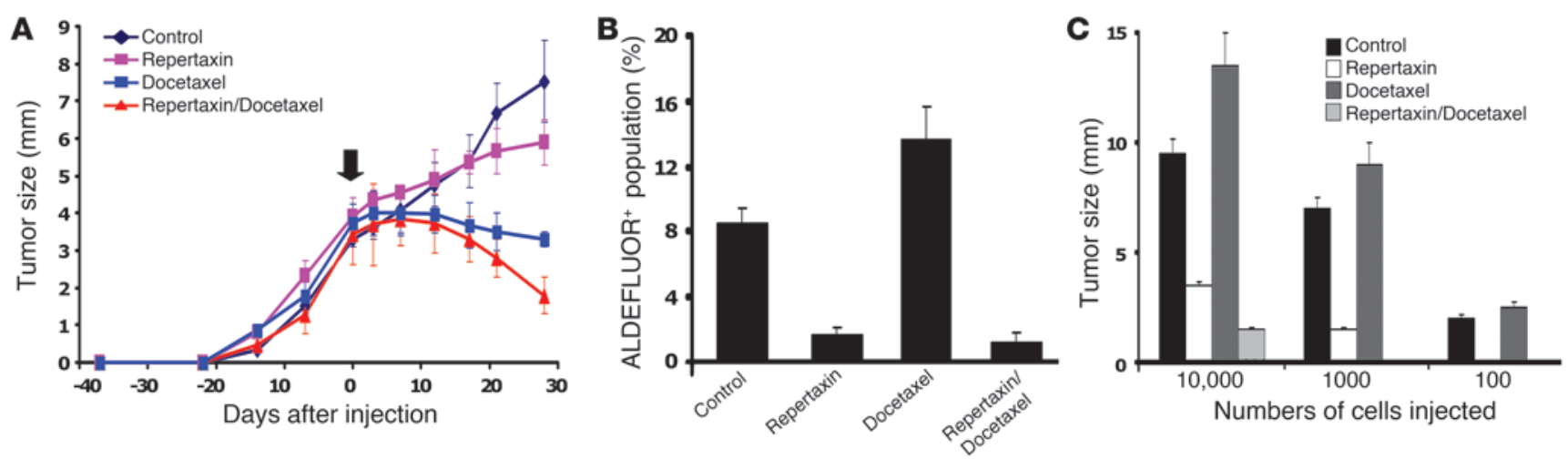

D

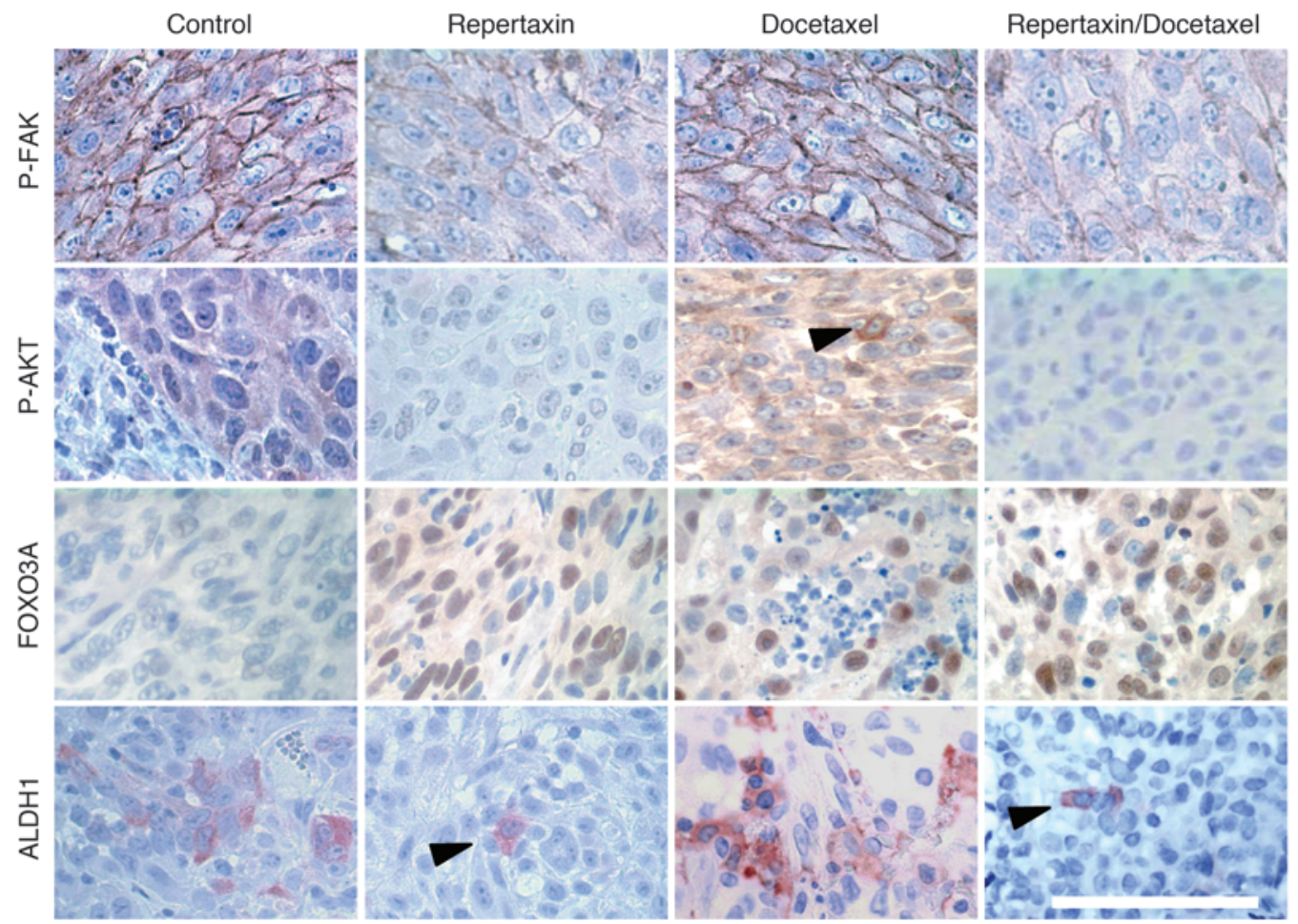

\section{Figure 4}

Effect of repertaxin treatment on the breast CSC population in vivo. (A-C) For each xenograft, 50,000 cells were injected into the mammary fat pad of mice. (A) Tumor size before and during the course of each indicated treatment (arrow indicates beginning of the treatment). Similar results were observed for each sample (UM2 shown here; see Supplemental Figure 12 for SUM159, MC1, and UM3), with a statistically significant size reduction of the tumor treated with docetaxel alone or in combination with repertaxin compared with the control tumors $(P<0.01)$. (B and C) Docetaxel-treated tumor showed similar or increase percentage of ALDEFLUOR ${ }^{+}$cells compared with the control, whereas repertaxin treatment alone or in combination produced a statistically significant decrease in ALDEFLUOR ${ }^{+}$cells $(P<0.01$; B $)$. Serial dilutions of cells obtained from these xenografts were implanted in the mammary fat pad of secondary mice, which received no further treatment. Cells from control and docetaxel-treated tumors formed secondary tumors at all dilutions, whereas only higher numbers of cells obtained from xenografts treated with repertaxin alone or in combination were able to generate tumors $(P<0.01 ; \mathbf{C})$. (D) Xenotransplants from each group were collected, and immunohistochemistry staining was done. Phospho-FAK, phospho-AKT, and ALDH1 expression was detected in the control and docetaxeltreated tumors, whereas low or no expression was detected in the tumors treated with repertaxin alone or in combination. Nuclear FOXO3A expression was detected in the cells treated with docetaxel and/or with repertaxin (arrowheads denote positive staining). Scale bar: $100 \mu \mathrm{m}$. Error bars represent mean \pm SD.

xenografts generated from 3 different patients (MC1, UM2, and UM3). Cells from these tumors were transplanted orthotopically into the humanized cleared fat pads of NOD/SCID mice, without cultivation in vitro. For each of these xenotransplants, we previously demonstrated that the CSC population was exclusively contained within the ALDEFLUOR ${ }^{+}$population $(12,13)$. In each of these tumors, we confirmed that the CXCR1 $1^{+}$population was almost exclusively contained within this ALDEFLUOR ${ }^{+}$population (Supplemental Table 1) and that the PTEN/FAK/AKT pathway was activated (Supplemental Figure 11). We injected 50,000 cells from each xenotransplant into the humanized fat pads of NOD/ SCID mice and monitored tumor growth. When the tumor size 
was approximately $4 \mathrm{~mm}$, we initiated treatment with repertaxin alone (15 mg/kg twice daily for 28 days), docetaxel alone $(10 \mathrm{mg} / \mathrm{kg}$ once weekly for 4 weeks), or a combination of both drugs. Tumor growth was compared with that of saline-injected controls. For each xenotransplant, we observed significant inhibition of tumor growth induced by treatment with docetaxel, alone or in combination with repertaxin (Figure 4A and Supplemental Figure 12). Repertaxin treatment alone had a moderate impact on tumor growth. After 4 weeks of treatment, animals were sacrificed, and the residual tumors were analyzed using the ALDEFLUOR assay. Residual tumors treated with docetaxel alone contained either an unchanged or an increased percentage of ALDEFLUOR ${ }^{+}$cells compared with untreated controls (Figure 4B and Supplemental Figure 12). This result is consistent with previous studies reporting no change or an expansion of the breast CSC population after chemotherapy $(6,7)$. In contrast, repertaxin treatment alone or in combination with docetaxel reduced the ALDEFLUOR ${ }^{+}$population by more than $75 \%$ (Figure 4B and Supplemental Figure 12). These observations were confirmed by immunohistochemistry of ALDH1 expression in the different xenotransplants. A decrease in ALDH1 ${ }^{+}$cells was detected in repertaxin-treated tumors compared with untreated tumors, whereas the percent of $\mathrm{ALDH} 1^{+}$cells was unchanged or increased in tumors treated with docetaxel alone (Figure 4D).

We also evaluated the presence of $\mathrm{CD} 44^{+} \mathrm{CD} 24^{-}$cells in these tumors. These markers have previously been shown to be expressed in breast CSCs (29). We first measured the overlap between the CD $44^{+} \mathrm{CD} 24^{-}$phenotype and CXCR1 expression. CXCR $1^{+}$cells were present both in the $\mathrm{CD} 44^{+} \mathrm{CD} 24^{-}$cell population and in the $\mathrm{CD} 24^{+}$or $\mathrm{CD} 44^{-}$populations (Supplemental Table 2 ). In residual tumors treated with docetaxel alone, we consistently observed either an unchanged or an increased percentage of CD $44^{+} \mathrm{CD} 24^{-}$ cells, whereas repertaxin treatment alone or in combination with docetaxel resulted in a reduction of the $\mathrm{CD} 44^{+} \mathrm{CD} 24^{-}$cell population (Supplemental Figure 13).

To further demonstrate that repertaxin treatment was able to reduce the CSC population, we used a functional in vivo assay consisting of reimplantation of cells from treated tumors into secondary NOD/SCID mice. This assay is a direct test assessing the tumor-initiating and self-renewal capacity of CSCs remaining after treatment. Tumor cells derived from control or docetaxeltreated animals showed similar tumor regrowth at all dilutions in secondary NOD/SCID mice. In contrast, repertaxin treatment with or without docetaxel reduced tumor growth in secondary recipients (Figure 4C). When equal numbers of cells were injected, those from repertaxin-treated animals showed a 2- to 5-fold reduction in tumor growth compared with cells from control or docetaxel-treated animals (Figure 4C). Moreover, for each xenotransplant model, 1,000 or 100 tumor cells obtained from animals treated with the combination of repertaxin and docetaxel failed to form any secondary tumors in NOD/SCID mice (Figure 4C, Supplemental Figure 12, and Supplemental Table 3). Together, these studies demonstrate that repertaxin treatment specifically targets and reduces the CSC population.

Repertaxin treatment inbibits FAK/AKT signaling and activates FOXO3A in vivo. To determine whether repertaxin treatment affected FAK/AKT signaling in vivo, we examined the expression of phospho-FAK and phospho-AKT by immunohistochemistry in each of the xenotransplants after treatment. Membranous phospho-FAK expression was detected in $50 \%$ of cells from the control and docetaxel-treated tumors, whereas phospho-FAK expression was abolished in the tumors treated with repertaxin alone or in combination with docetaxel (Figure 4D). Similar results were observed for the phospho-AKT expression, with $70 \%$ of cells expressing phospho-AKT in the untreated tumors, $20 \%$ phosphoAKT-positive cells in docetaxel-treated tumors, and complete inhibition of phospho-AKT expression in the tumors treated with repertaxin alone or in combination with docetaxel (Figure 4D). Interestingly, nuclear FOXO3A was detected in the cells from the tumors treated with docetaxel alone, repertaxin alone, and the combination. These in vivo data are consistent with the in vitro data and confirm that repertaxin treatment inhibits FAK/AKT signaling and activates FOXO3A.

Repertaxin treatment reduces the development of systemic metastasis. To determine whether repertaxin reduces systemic metastasis, we infected HCC1954, MDA-MB-453, and SUM159 breast cancer cell lines with a luciferase lentivirus reporter system and introduced the cells into NOD/SCID mice by intracardiac injection. A suspension of 250,000 cells per cell line was injected, and metastasis formation was monitored once per week by bioluminescent imaging. At 12 hours after intracardiac injection, mice were injected twice daily with repertaxin or saline control. As shown in Figure 5, A and B, repertaxin treatment significantly reduced metastasis formation from HCC1954 and SUM159 cells, as determined by photon flux emission. Only 1 animal of 6 developed metastasis in the repertaxin-treated group, whereas 5 of 6 animals developed metastasis in the control group $(P<0.05)$. Histologic sections confirmed the presence of metastases at several sites in untreated animals (Figure 5D). Interestingly, repertaxin treatment did not have any effect on metastasis formation in mice injected with MDA-MB-453 cells (Figure 5C). The photon flux emission and the number of animals that developed metastasis were similar in the repertaxin-treated and untreated groups. This result is consistent with our above data that described MDA-MB-453 as a cell line resistant to repertaxin due to the presence of a PTEN mutation. Taken together, these results suggest that CXCR1 blockade with agents such as repertaxin may be able to reduce metastasis which is mediated by the CSC population (13).

\section{Discussion}

Accumulating evidence suggests that cellular subcomponents with stem cell properties may drive tumor growth and metastasis (1). Furthermore, by virtue of their relative resistance to current therapeutic modalities, these cells may contribute to treatment resistance and relapse (2). If this is the case, then the development of strategies to effectively target the CSC population will be required to improve therapeutic outcome. We describe such an approach based on blocking the CXCR1 cytokine receptor, which is expressed on breast CSCs. Experiments in a number of systems have demonstrated that cytokine networks play an important role in tumorigenesis. Furthermore, there is evidence that several of these cytokines may regulate stem cell behavior. IL-4 is capable of regulating self-renewal of pancreatic CSCs (30), and IL-6 can regulate CSCs in colon and breast cancer (31). The role of IL-8 in mediating tumor invasion and metastasis has previously been demonstrated $(14,21)$. In addition, IL-8 increases neural stem cell self-renewal during wound healing in the brain (32). Recently, lung CSCs were described as expressing the chemokine receptor CXCR1 (33). Consistent with these findings, we demonstrated that the CXCR1 $1^{+}$population was almost exclusively contained within the ALDEFLUOR ${ }^{+}$population in breast cancer cell lines and primary xenografts as well as in normal 
A

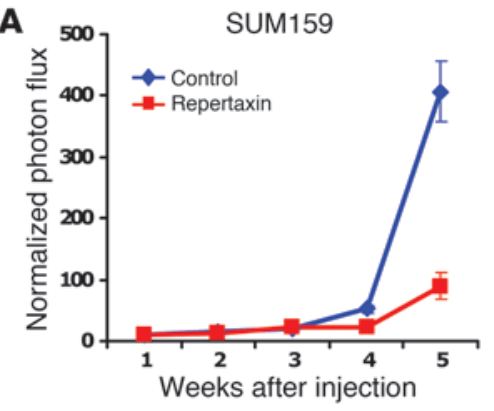

Control

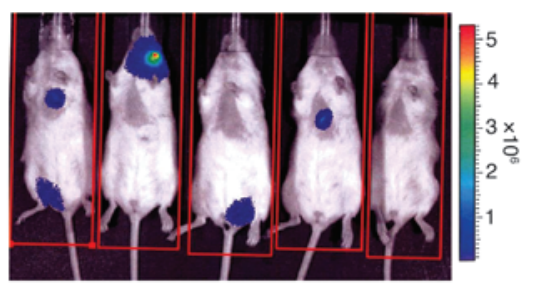

Repertaxin

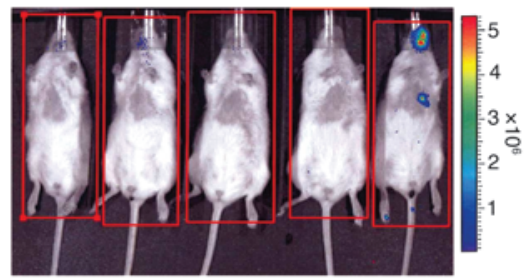

B

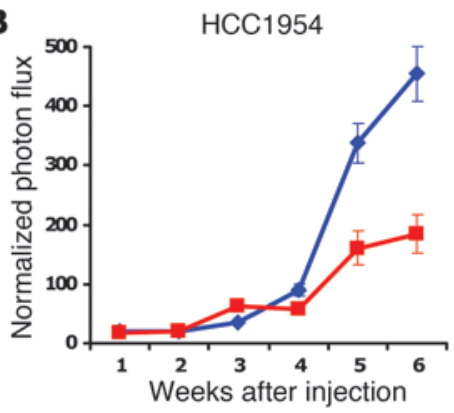

Control

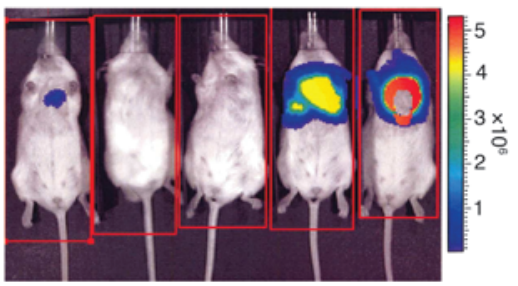

Repertaxin

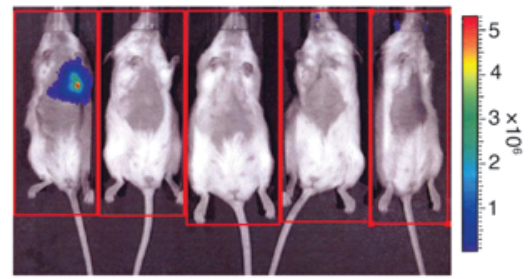

C

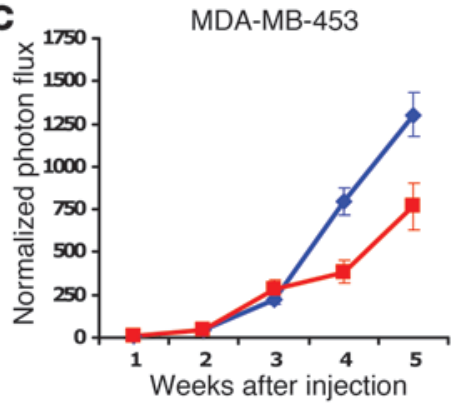

Control
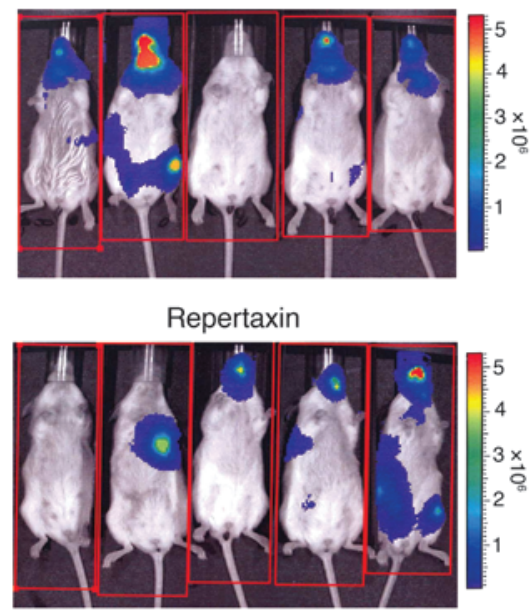

D

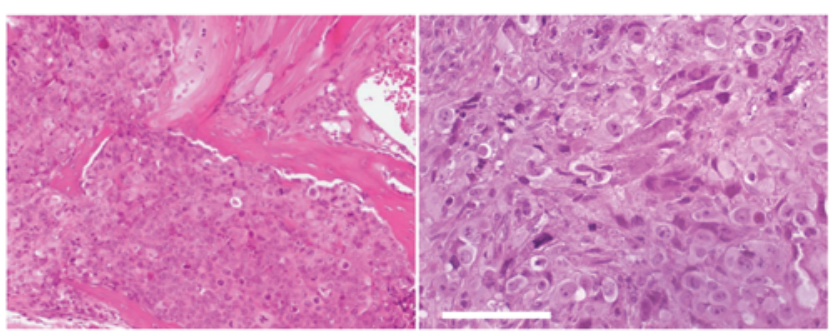

Figure 5

Repertaxin treatment reduces the development of systemic metastasis. (A-C) To evaluate the effect of repertaxin treatment on metastasis formation, we infected HCC1954, SUM159, and MDA-MB-453 breast cancer cell lines with a lentivirus expressing luciferase, and inoculated 250,000 luciferase-infected cells into NOD/SCID mice via intracardial injection. Mice were treated 12 hours after intracardiac injection with either s.c. injection of saline solution or s.c. injection of $15 \mathrm{mg} / \mathrm{kg}$ repertaxin twice daily for 28 days. Metastasis formation was monitored using bioluminescence imaging. Quantification of the normalized photon flux, measured at weekly intervals following inoculation, revealed a statistically significant decrease $(P<0.01)$ in metastasis formation in repertaxin-treated compared with saline controls for mice inoculated with $\mathrm{HCC} 1954$ (A) or SUM159 (B) cells. In contrast, repertaxin treatment did not have any effect on metastasis formation for the mice injected with MDA-MB453 (C) cells. (D) Histologic confirmation, by H\&E staining, of metastasis in bone and soft tissue resulting from mice not treated with repertaxin. Scale bar: $100 \mu \mathrm{m}$. Error bars represent mean \pm SD.

human mammary cells. We found that this chemokine receptor was overexpressed in ALDEFLUOR ${ }^{+}$breast cancer cell populations (13). In breast cancers, IL-8 is produced in the tumor microenvironment by a number of cell types, including inflammatory cells, vascular endothelial cells, tumor-associated fibroblasts, and mesenchymal stem cells (21). Because cytokine networks may mediate interaction among these cell types, we sought to target CSCs through blockade of the IL-8 receptor CXCR1.

Using in vitro assays, we demonstrated that blockade of CXCR1, but not of the alternative IL-8 receptor CXCR2, reduced the breast
CSC population. This was followed by induction of apoptosis in the entire remaining cell population, which lacks CXCR1 expression. In addition to CXCR1-blocking antibodies, we demonstrated that repertaxin, a CXCR $1 / 2$ inhibitor, induced similar effects by targeting the $\mathrm{CXCR} 1^{+}$population. In contrast to its direct effects on the CXCR1-expressing CSC population, repertaxin had no direct effect on the bulk tumor cell population, which lacks CXCR1 expression. This suggested that CXCR1 blockade in CXCR $1^{+}$cells induced cell death in CXCR1- cells via a bystander effect. We confirmed this hypothesis and identified the FASL/FAS pathway as 
A

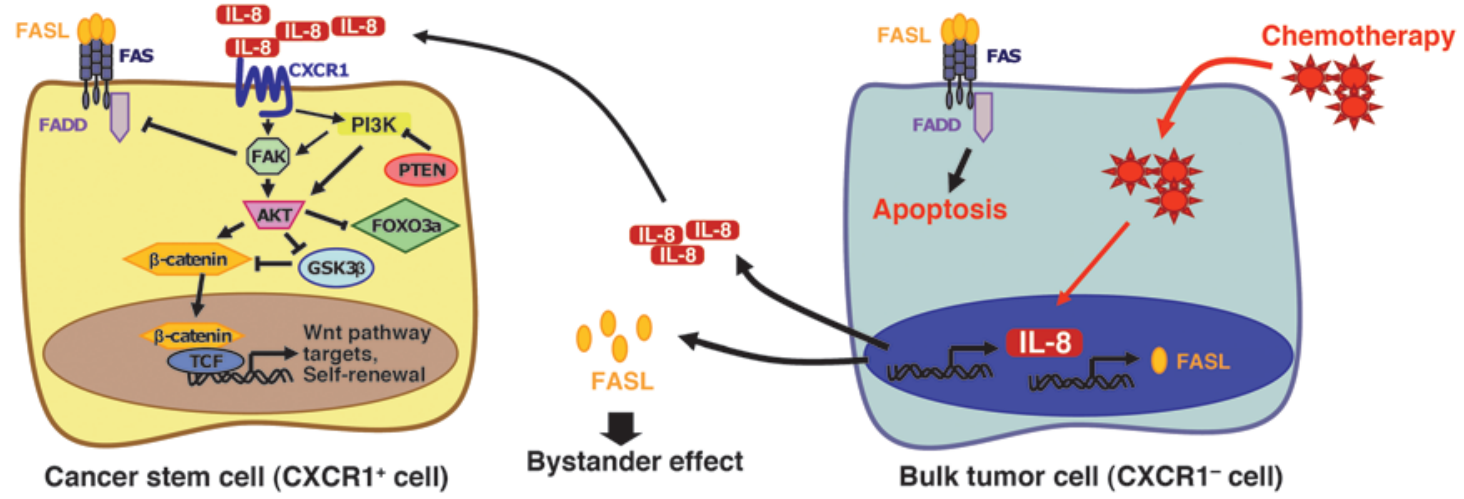

B

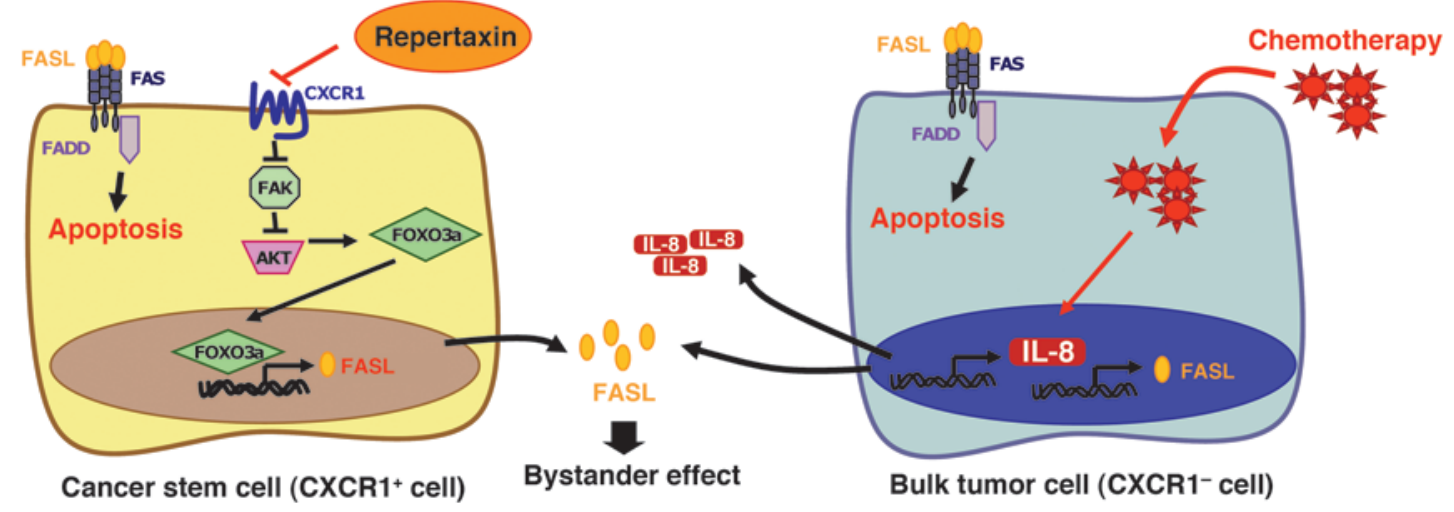

Figure 6

IL-8/CXCR1 signaling in CSCs treated with chemotherapy alone or in combination with repertaxin. (A) Potential IL-8/CXCR1 cell signaling in CSCs. CXCR1 activation upon IL-8 binding induces FAK phosphorylation. Active FAK phosphorylates AKT and activates the WNT pathway, which regulates stem cell self-renewal and FOXO3A that regulates cell survival. Activation of FAK protects CSCs from a FASL/FAS-mediated bystander effect by inhibiting FADD, a downstream effector of FAS signaling. In the presence of chemotherapy, only the bulk tumor cells are sensitive to the treatment and release a high level of IL-8 and FASL proteins during the apoptotic process. Breast CSCs are stimulated via an IL-8-mediated bystander effect and are resistant to the bystander killing effect mediated by FASL. TCF, T cell factor. (B) Repertaxin treatment blocks IL-8/CXCR1 signaling and inhibits breast CSC self-renewal and survival. When repertaxin treatment is combined with chemotherapy, the CSCs are sensitized to the bystander killing effect mediated by FASL.

the mediator of this bystander killing effect. This phenomenon explains the efficacy of repertaxin treatment in inducing massive apoptosis in the entire cell population despite the fact that the CXCR $1^{+}$population represents less than $1 \%$ of the cell population. The role of FASL was demonstrated by the effective blocking of bystander killing by anti-FASL antibody.

The FASL/FAS death pathway may play a role in normal mammary physiology as well as in mediating chemotherapy-induced cell death. In the normal breast, the rapid decrease in hormone levels after lactation leads to massive apoptosis in the mammary gland that is largely mediated by FASL-induced FAS apoptosis (19). Interestingly, involuting differentiated mammary cells undergoing apoptosis also secrete increased levels of IL-8 (19). Based on our current studies, we speculate that this secreted IL-8 interacts with CXCR1 on normal mammary stem cells to increase their selfrenewal as well as protecting them from FAS-mediated apoptosis. This process ensures sufficient regeneration of the mammary stem cell pool to support future pregnancy/lactation cycles.

Similar cytokine interactions may occur in tumors exposed to cytotoxic chemotherapy. Chemotherapy may directly induce cellular apoptosis in differentiated tumor cells as well as induce the production of FASL by these dying cells that in turn induces apoptosis in surrounding tumor cells via a FAS-mediated bystander effect. However, concomitant with the production of FASL, these injured cells also secrete increased levels of IL-8 in a process resembling mammary involution or wound healing. As is the case in the involuting mammary gland, this IL-8 may stimulate breast CSCs as well as protecting them from apoptosis. This may contribute to the relative increase in CSCs observed after chemotherapy in preclinical models (6) and neoadjuvant clinical trials (7-9). The effects of chemotherapy on apoptosis and self-renewal pathways in tumors are shown in Figure 6.

To determine whether CXCR1 blockade could target breast CSCs in vivo, we compared the effects of the cytotoxic agent docetaxel with those of repertaxin on the CSC compartment and on tumor growth in NOD/SCID mice. We chose docetaxel because it is one of the most effective chemotherapeutic agents currently used to treat women with breast cancer. We assessed the CSC populations by the ALDEFLUOR assay and by serial transplantation in NOD/SCID mice. Using these assays, we determined that chemotherapy treatment alone resulted in either no change or a relative increase in the CSC populations. In con- 
trast, repertaxin treatment alone or in combination with chemotherapy significantly reduced the CSC population. Despite the significant reduction in the tumor-initiating populations, use of repertaxin alone did not result in a significant reduction in tumor size. This suggests that, unlike its in vitro effects, use of repertaxin in vivo failed to induce significant bystander killing in the bulk nontumor population. This may be caused by survival signals generated by the tumor microenvironment in vivo. Nevertheless, the combination of repertaxin plus chemotherapy resulted in significant reductions in tumor size and in the CSC population, which suggests that a strategy of combining these agents to target both CSCs and bulk tumor cell populations may maximize the efficacy of these treatments.

To elucidate the mechanism of action of repertaxin, we analyzed the pathways downstream from CXCR1. CXCR1 has previously been shown to act through the FAK/AKT signaling pathway (21). We confirmed the interaction among CXCR1, FAK, and AKT and demonstrated that CXCR1 blockade acted specifically through FAK and AKT activation. We have previously shown that AKT activation regulates normal and malignant breast stem cell selfrenewal through phosphorylation of GSK3 $\beta$, resulting in the activation of the WNT pathway (26). This may explain why cells with PTEN knockdown were resistant to repertaxin. An additional important function of AKT is the regulation of cell survival through phosphorylation of FOXO3A. AKT phosphorylation of FOXO3A results in its cytoplasmic sequestration. In contrast, we demonstrated that CXCR1 blockade led to decreased AKT activation, which results in the translocation of FOXO3A in the nucleus and subsequent induction of a number of genes, including FASL (24). FASL induced via CXCR1 blockade is in turn responsible for the bystander killing effects that we observed in the present study. These pathways are illustrated in Figure 6.

In addition to its role in CXCR1 signaling, FAK mediates the interactions of cells with extracellular matrix components through integrin receptors (21). We previously demonstrated a role for FAK signaling in regulating the self-renewal of normal and malignant mouse mammary stem cells in transgenic models (34). FAK activation also promotes cell survival by blocking apoptosis mediated by FAS-associated protein with death domain (FADD) and receptorinteracting protein (RIP) $(35,36)$. This may explain the resistance of the CSC population to the FASL/FAS-induced apoptosis.

We and others have previously demonstrated that breast CSCs may play an important role in tumor invasion and metastasis (13, 37). Furthermore, there is evidence that IL- 8 and CXCR 1 also play important roles in these processes. Therefore, we analyzed the effects of CXCR1 blockade using repertaxin on the formation of experimental metastasis. We demonstrated that CXCR1 blockade reduced the development of metastasis when administered after intracardiac injection of breast cancer cells.

IL-8, in addition to other cytokines such has IL-6, has been implicated in tissue inflammation and repair as well as carcinogenesis (21). In fact, repertaxin was originally developed to block IL-8 activity to reduce tissue damage after myocardial infarction or stroke (15). Clinical phase I studies using this compound have demonstrated a lack of toxicity. This suggests that strategies aimed at interfering with cytokine regulatory loops such as IL-8 and CXCR1 may represent a novel strategy to target breast CSCs. Because these cells may drive tumor progression and metastasis, such strategies may lead to improving outcomes for women with advanced breast cancer.

\section{Methods}

Study approval. Use of anonymous human tissue samples was exempted from institutional review board approval by the NIH Office of Human Subjects Research. Animal studies were approved by the University of Michigan Unit for Laboratory Animal Medicine.

Dissociation of mammary tissue. Normal breast tissue from reduction mammoplasties (100-200 g) was minced with scalpels and dissociated enzymatically, and single cells were cultured in suspension to generate mammospheres or on a collagen substratum in adherent condition to induce cellular differentiation, as described previously (20).

Cell culture. Breast cancer cell lines HCC1954 and MDA-MB-453 were obtained from the ATCC, and line SUM159 was obtained from S. Ethier (Karmanos Cancer Institute, Detroit, Michigan, USA). The cell lines were grown using the recommended culture conditions (9). Breast cancer cell lines were treated in adherent condition with repertaxin (SigmaAldrich), anti-human CXCR1 mouse monoclonal antibody (clone 42705; R\&D Systems), anti-human CXCR2 mouse monoclonal antibody (clone 48311; R\&D Systems), anti-human CD95 mouse monoclonal antibody (clone DX2; BD Biosciences - Pharmingen) used as a FAS signaling agonist, anti-human FASL mouse monoclonal antibody (clone NOK-1; BD Biosciences - Pharmingen) used as a FAS signaling antagonist, or with docetaxel (Taxotere; Sanofi-Aventis).

Cell viability. For MTT assays, cells were plated in adherent condition in 96-well plates at 5,000 cells per well. After 1 day, treatment with repertaxin was started. The effect of repertaxin treatment on cell viability was estimated at different time points by addition of $20 \mu \mathrm{l}$ MTT solution $(5 \mathrm{mg} / \mathrm{ml}$ in PBS) in each well. Cells were then incubated for 1 hour at $37^{\circ} \mathrm{C}$, followed by addition of $50 \mu \mathrm{l}$ DMSO to each well. Absorbance was measured at $560 \mathrm{~nm}$ in a fluorescence plate reader (Spectrafluor; Tecan). For TUNEL assays, cells were plated in adherent conditions in 6-well plates at 50,000 cells per well. After 1 day, treatment with repertaxin was started. The number of apoptotic cells was estimated after 4 days of treatment. Cells were fixed in 3.7\% formaldehyde and stained using the TACS TdT kit (R\&D Systems). Nuclei were counterstained with DAPI/ antifade (Invitrogen). Sections were examined with a fluorescent microscope (Leica), with apoptotic cells detected in green.

ALDEFLUOR assay. The ALDEFLUOR kit (StemCell Technologies) was used to isolate the population with high ALDH enzymatic activity using a FACStarPLUS (BD) as previously described (12). In order to eliminate cells of mouse origin from the xenotransplanted tumors, we used staining with an anti-H2Kd antibody (diluted 1:200, 20 minutes on ice; BD Biosciences) followed by staining with a secondary antibody labeled with PE (diluted 1:250, 20 minutes on ice; Jackson Labs).

ELISA assay. To measure the level of soluble FASL secreted in the culture medium of cells treated or not with repertaxin, we used the Human sFAS Ligand Elisa (Bender Medsystems). Absorbance was read on a spectrophotometer using $450 \mathrm{~nm}$ as the primary wavelength. Each measurement was done in quadruplicate.

Western blotting. Cells were lysed in a Laemmli buffer and loaded onto SDS-polyacrylamide gels. Blots were incubated with the respective primary antibodies diluted in TBST (containing $0.1 \%$ Tween 20 and $2 \%$ BSA) either overnight at $4^{\circ} \mathrm{C}$ or for 2 hours at room temperature. Then, blots were washed and incubated with appropriate secondary antibodies (GE Healthcare) and detected using SuperSignal West Pico Chemiluminescent Substrate (Pierce).

Immunostaining. For immunofluorescent staining, sorted CXCR $1^{+}$cells were fixed with $95 \%$ methanol at $-20^{\circ} \mathrm{C}$ for 10 minutes. Cells were rehydrated in PBS and incubated with respective antibodies at room temperature for 1 hour. Primary antibodies used were P-FAK (diluted 1:50; Cell Signaling Technology), P-AKT (diluted 1:300; Cell Signaling Technology), and FOXO3A 
(diluted 1:250; Cell Signaling Technology). Slides were then washed and incubated for 30 minutes with PE-conjugated secondary antibodies (Jackson Labs). The nuclei were counterstained with DAPI/antifade (Invitrogen) and coverslipped. Sections were examined with a fluorescent microscope (Leica). Immunohistochemistry for the detection of ALDH1 (diluted 1:100; BD Biosciences), P-FAK, P-AKT, and FOXO3A expression was done on paraffin section as previously described (38). Staining was done using the Histostainplus kit (Zymed Laboratories). DAB or 3-amino-9-ethylcarbazole was used as chromogen, and sections were counterstained with hematoxylin.

Animalmodel. Tumorigenicity of ALDEFLUOR ${ }^{+} \mathrm{CXCR} 1^{+}$and ALDEFLUOR ${ }^{+}$ CXCR1- SUM159 cells was assessed in NOD/SCID mice. Fat pads were prepared as described previously (12). To explore the efficiency of repertaxin treatment on tumor growth, we used the SUM159 cell line and 3 primary human breast cancer xenografts generated from 3 different patients (MC1, UM2, and UM3; ref. 12). Cells from these tumors were transplanted orthotopically in the humanized cleared fat pad of NOD/SCID mice, without cultivation in vitro. We injected 50,000 cells from each xenotransplant in the humanized fat pad of NOD/SCID mice and monitored the tumor growth. When the tumor size was approximately $4 \mathrm{~mm}$, we initiated treatment with repertaxin alone (15 $\mathrm{mg} / \mathrm{kg}$ s.c. twice daily for 28 days), docetaxel alone (10 mg/kg i.p. once weekly for 4 weeks), repertaxin and docetaxel in combination, or a control group injected with saline (once weekly i.p. and twice daily s.c. for 28 days). We injected 5 mice for each xenotransplant and for each group. The animals were euthanized when the tumors were approximately $1.5 \mathrm{~cm}$ in the largest diameter, to avoid tumor necrosis and in compliance with regulations for use of vertebrate animals in research. A portion of each fat pad injected was fixed in formalin and embedded in paraffin for histological analysis. The rest of the tumor cells were reimplanted into secondary NOD/SCID mice. Serial dilutions of cells were used for the reimplantation with injection of 10,000, 1,000, and 100 cells for each treated tumor.

Anchorage-independent culture. Breast cancer cell lines, treated in adherent conditions with repertaxin $(100 \mathrm{nM})$, anti-CXCR1 antibody $(10 \mu \mathrm{g} / \mathrm{ml})$, or anti-CXCR2 antibody $(10 \mu \mathrm{g} / \mathrm{ml})$, were dissociated and plated as single cells in ultralow attachment plates (Corning) at low density (5,000 viable cells $/ \mathrm{ml}$ ). Cells were grown as previously described (13). Subsequent cultures after dissociation of primary tumorspheres were plated on ultralow attachment plates at a density of 5,000 viable cells $/ \mathrm{ml}$. The capacity of cells to form tumorspheres was quantified after the first (primary tumorspheres) and second (secondary tumorspheres) passage.

RNA extraction and real-time quantitative RT-PCR. After SUM159 cells were treated, total RNA was isolated using RNeasy Mini Kit (QIAGEN) and used for real-time quantitative RT-PCR assays in a ABI PRISM 7900HT sequence detection system. Primers and probes for the Taqman system were selected from the Applied Biosystems Web site (FASL assay ID, Hs_00899442_mi; IL8 assay ID, Hs_00174103_mi, TBP assay ID, Hs_00427620_mi). The relative expression mRNA level of FASL and IL8 was computed with respect to the internal standard TBP gene to normalize for variations in the quality of RNA and the amount of input cDNA, as described previously (39).

Flow cytometry analysis. Staining for CD44, CD24, and Lin was performed as previously described (12). Staining for CD95 and FAS was performed using an APC-labeled anti-CD95 antibody (diluted 1:20; BD Biosciences).
For CXCR1 and CXCR2 staining, we used primary antibodies anti-CXCR1 (diluted 1:100, clone 42705; R\&D Systems) and anti-CXCR2 (diluted 1:100, clone 48311; R\&D Systems) followed by staining with a secondary PE-labeled anti-mouse antibody (diluted 1:250; Jackson Labs). Fresh cells were stained with $1 \mu \mathrm{g} / \mathrm{ml}$ PI (Sigma-Aldrich) for 5 minutes for viability.

Virus infection. We produced 2 different lentiviral constructs, for the expression of Luciferase gene (Lenti-LUC-VSVG; ref. 13) and for the inhibition PTEN expression (Lenti-PTEN-SiRNA-DsRed; ref. 26). All lentiviral constructs were prepared by the University of Michigan Vector. We also used an adenoviral construct for the overexpression of FAK (Ad-FAK-GFP; ref. 34) generated in our laboratory. Cells infection with different vectors was performed as previously described (13). Efficiency of infection was verified by measuring the percentage of DsRed- or GFP-expressing cells.

Intracardiac inoculation. NOD/SCID mice ( 6 weeks old) were anesthetized with a $2 \%$ isoflurane/air mixture and injected in the heart left ventricle with 250,000 cells in $100 \mu \mathrm{l}$ sterile Dulbecco PBS lacking $\mathrm{Ca}^{2+}$ and $\mathrm{Mg}^{2+}$. For each of the cell lines HCC1954, MDA-MB-453, and SUM159, and for each treatment, saline or repertaxin, 6 animals were injected. At 12 hours after intracardiac injections, mice were begun on twice-daily injection with repertaxin or saline (control).

Bioluminescence detection. Baseline bioluminescence was assessed before inoculation and each week thereafter. Bioluminescence detection procedures were performed as previously described (13). Normalized photon flux represents the ratio of the photon flux detected each week after inoculation to that detected before inoculation.

Statistics. Results are presented as mean \pm SD for at least 3 repeated individual experiments for each group. Statistical analyses used SPSS software, version 10.0.5. Correlations between sample groups and molecular parameters were calculated by Fisher exact test or 1-way ANOVA for independent samples. A $P$ value less than 0.05 was considered significant.

\section{Acknowledgments}

Thanks are due to the University of Michigan Cancer Center Flow Cytometry core. This work was supported by NIH grants CA129765 and CA101860, The Breast Cancer Foundation, the Taubman Institute, the University of Michigan Cancer Center NIH support grant 5P30CA46592, INSERM, Institut Paoli-Calmettes, Institut National du Cancer grants DHOS2008 and ACI2007, and Ligue Nationale Contre le Cancer grant Label DB.

Received for publication March 31, 2009, and accepted in revised form November 11, 2009.

Address correspondence to: Max S. Wicha, University of Michigan Comprehensive Cancer Center, Int Med-Hematology/Oncology, 1500 E. Medical Center Drive, Ann Arbor, Michigan 48109-0015, USA. Phone: (734) 647-9923; Fax: (734) 647-9480; E-mail: mwicha @umich.edu. Or to: Christophe Ginestier, Centre de Recherche en Cancérologie de Marseille, U891/Inserm/Institut Paoli-Calmettes, Laboratoire d'Oncologie Moleculaire, 27 Bd Lei Roure, 13009 Marseille, France. Phone: 33-4-91-22-35-09; Fax: 33-4-91-22-35-44; E-mail: christophe.ginestier@inserm.fr.
1. Visvader JE, Lindeman GJ. Cancer stem cells in solid tumours: accumulating evidence and unresolved questions. Nat Rev Cancer. 2008;8(10):755-768.

2. Reya T, Morrison SJ, Clarke MF, Weissman IL. Stem cells, cancer, and cancer stem cells. Nature. 2001; 414(6859):105-111.

3. Quintana E, Shackleton M, Sabel MS, Fullen DR, Johnson TM, Morrison SJ. Efficient tumour formation by single human melanoma cells. Nature.
2008;456(7222):593-598.

4. Kelly PN, Dakic A, Adams JM, Nutt SL, Strasser A. Tumor growth need not be driven by rare cancer stem cells. Science. 2007;317(5836):337.

5. Phillips TM, McBride WH, Pajonk F. The response of CD24(-/low)/CD44+ breast cancer-initiating cells to radiation. J Natl Cancer Inst. 2006;98(24):1777-1785.

6. Yu F, et al. let-7 regulates self renewal and tumorigenicity of breast cancer cells. Cell. 2007;
131(6):1109-1123.

7. Li X, et al. Intrinsic resistance of tumorigenic breast cancer cells to chemotherapy. J Natl Cancer Inst. 2008; 100(9):672-679.

8. Creighton CJ, et al. Residual breast cancers after conventional therapy display mesenchymal as well as tumor-initiating features. Proc Natl Acad SciUS A. 2009;106(33):13820-13825.

9. Tanei $\mathrm{T}$, et al. Association of breast cancer stem cells 
identified by aldehyde dehydrogenase 1 expression with resistance to sequential Paclitaxel and epirubicin-based chemotherapy for breast cancers. Clin Cancer Res. 2009;15(12):4234-4241.

10. Korkaya H, Wicha MS. Selective targeting of cancer stem cells: a new concept in cancer therapeutics. BioDrugs. 2007;21(5):299-310.

11. Brisken C, Duss S. Stem cells and the stem cell niche in the breast: an integrated hormonal and developmental perspective. Stem Cell Rev. 2007; 3(2):147-156.

12. Ginestier C, et al. ALDH1 is a marker of normal and malignant human mammary stem cells and a predictor of poor clinical outcome. Cell Stem Cell. 2007; 1(5):555-567.

13. Charafe-Jauffret E, et al. Breast cancer cell lines contain functional cancer stem cells with metastatic capacity and a distinct molecular signature. Cancer Res. 2009;69(4):1302-1313.

14. Inoue $\mathrm{K}$, et al. Interleukin 8 expression regulates tumorigenicity and metastases in androgen-independent prostate cancer. Clin Cancer Res. 2000; 6(5):2104-2119.

15. Bertini R, et al. Noncompetitive allosteric inhibitors of the inflammatory chemokine receptors CXCR1 and CXCR2: prevention of reperfusion injury. Proc Natl Acad Sci U S A. 2004;101(32):11791-11796.

16. Ponti $\mathrm{D}$, et al. Isolation and in vitro propagation of tumorigenic breast cancer cells with stem/progenitor cell properties. Cancer Res. 2005;65(13):5506-5511.

17. Hollestelle A, Elstrodt F, Nagel JH, Kallemeijn WW, Schutte M. Phosphatidylinositol-3-OH kinase or RAS pathway mutations in human breast cancer cell lines. Mol Cancer Res. 2007;5(2):195-201.

18. Chhipa RR, Bhat MK. Bystander killing of breast cancer MCF-7 cells by MDA-MB-231 cells exposed to 5 -fluorouracil is mediated via Fas. J Cell Biochem. 2007;101(1):68-79.
19. Song J, et al. Roles of Fas and Fas ligand during mammary gland remodeling. J Clin Invest. 2000; 106(10):1209-1220.

20. Dontu G, et al. In vitro propagation and transcriptional profiling of human mammary stem/progenitor cells. Genes Dev. 2003;17(10):1253-1270.

21. Waugh DJ, Wilson C. The interleukin-8 pathway in cancer. Clin Cancer Res. 2008;14(21):6735-6741.

22. Vivanco I, Sawyers CL. The phosphatidylinositol 3-Kinase AKT pathway in human cancer. Nat Rev Cancer. 2002;2(7):489-501.

23. Brunet A, et al. Protein kinase SGK mediates survival signals by phosphorylating the forkhead transcription factor FKHRL1 (FOXO3a). Mol Cell Biol. 2001;21(3):952-965.

24. Jonsson H, Allen P, Peng SL. Inflammatory arthritis requires Foxo3a to prevent Fas ligand-induced neutrophil apoptosis. Nat Med. 2005;11(6):666-671.

25. Dubrovska A, et al. The role of PTEN/Akt/PI3K signaling in the maintenance and viability of prostate cancer stem-like cell populations. Proc Natl Acad Sci US A. 2009;106(1):268-273.

26. Korkaya H, et al. Regulation of mammary stem/ progenitor cells by PTEN/Akt/beta-catenin signaling. PLoS Biol. 2009;7(6):e1000121.

27. Yilmaz $\mathrm{OH}$, et al. Pten dependence distinguishes haematopoietic stem cells from leukaemia-initiating cells. Nature. 2006;441(7092):475-482.

28. Schaub FJ, et al. Fas/FADD-mediated activation of a specific program of inflammatory gene expression in vascular smooth muscle cells. Nat Med. 2000;6(7):790-796.

29. Al Hajj M, Wicha MS, Benito-Hernandez A, Morrison SJ, Clarke MF. Prospective identification of tumorigenic breast cancer cells. Proc Natl Acad Sci U S A. 2003;100(7):3983-3988.

30. Todaro $\mathrm{M}$, et al. Colon cancer stem cells dictate tumor growth and resist cell death by production of interleukin-4. Cell Stem Cell. 2007;1(4):389-402.

31. Sansone P, et al. IL-6 triggers malignant features in mammospheres from human ductal breast carcinoma and normal mammary gland. J Clin Invest. 2007;117(12):3988-4002.

32. Beech JS, et al. The MHP36 line of murine neural stem cells expresses functional CXCR1 chemokine receptors that initiate chemotaxis in vitro. J Neuroimmunol. 2007;184(1-2):198-208.

33. Levina $V$, et al. Drug-selected human lung cancer stem cells: cytokine network, tumorigenic and metastatic properties. PLoS ONE. 2008;3(8):e3077.

34. Luo M, et al. Mammary epithelial-specific ablation of the focal adhesion kinase suppresses mammary tumorigenesis by affecting mammary cancer stem/ progenitor cells. Cancer Res. 2009;69(2):466-474.

35. Kurenova E, et al. Focal adhesion kinase suppresses apoptosis by binding to the death domain of receptor-interacting protein. Mol Cell Biol. 2004; 24(10):4361-4371.

36. Xu LH, et al. The focal adhesion kinase suppresses transformation-associated, anchorage-independent apoptosis in human breast cancer cells. Involvement of death receptor-related signaling pathways. J Biol Chem. 2000;275(39):30597-30604.

37. Croker AK, et al. High aldehyde dehydrogenase and expression of cancer stem cell markers selects for breast cancer cells with enhanced malignant and metastatic ability [published online ahead of print August 4, 2008]. J Cell Mol Med. doi:10.1111/ j.1582-4934.2008.00455.x.

38. Ginestier C, et al. Distinct and complementary information provided by use of tissue and DNA microarrays in the study of breast tumor markers. Am J Pathol. 2002;161(4):1223-1233.

39. Ginestier C, et al. Prognosis and gene expression profiling of 20q13-amplified breast cancers. Clin Cancer Res. 2006;12(15):4533-4544. 